\title{
BMJ Open Non-aristolochic acid prescribed Chinese herbal medicines and the risk of mortality in patients with chronic kidney disease: results from a population-based follow-up study
}

\author{
Chuan Fa Hsieh, ${ }^{1,2}$ Song Lih Huang, ${ }^{3}$ Chien Lung Chen, ${ }^{4}$ Wei Ta Chen, ${ }^{5}$ Huan \\ Cheng Chang, ${ }^{4,6}$ Chen Chang Yang ${ }^{1,7}$
}

To cite: Hsieh CF, Huang SL, Chen CL, et al. Nonaristolochic acid prescribed Chinese herbal medicines and the risk of mortality in patients with chronic kidney disease: results from a population-based follow-up study. BMJ Open 2014;4: e004033. doi:10.1136/ bmjopen-2013-004033

- Prepublication history for this paper is available online. To view these files please visit the journal online (http://dx.doi.org/10.1136/ bmjopen-2013-004033).

Received 15 September 2013 Revised 29 January 2014 Accepted 2 February 2014

CrossMark

For numbered affiliations see end of article.

Correspondence to Dr Chen-Chang Yang; ccyang@vghtpe.gov.tw

\section{ABSTRACT}

Objectives: To evaluate the relationship between the use of non-aristolochic acid (AA) prescribed Chinese herbal medicines (CHMs) and the risk of mortality in patients with chronic kidney disease (CKD).

Design: Nationwide population-based follow-up study.

Setting: Longitudinal health insurance database sampled from the Taiwan National Health Insurance Research Database.

Participants: A total of 47876 patients with CKD were identified. Participants who had ever used AA-containing $\mathrm{CHMs}$, had cancer or HIV prior to the diagnosis of CKD, died within the first month of CKD diagnosis and who were not Taiwanese citizens were excluded. A total of 13864 participants were eligible for final analysis.

Primary and secondary outcome measures: Allcause mortality among patients with CKD between 2000 and 2008.

Results: After controlling for potential confounders, we found that participants who started to receive nonAA prescribed CHMs after the diagnosis of CKD had a lower risk of mortality as compared with non-users of non-AA prescribed CHMs (adjusted HR (aHR) 0.6; $95 \% \mathrm{Cl} 0.4$ to $0.7, p<0.001$ ). Moreover, participants who had used non-AA prescribed CHMs prior to and after the diagnosis of CKD also had a lower risk of mortality than non-users (aHR $0.6 ; 95 \% \mathrm{Cl} 0.5$ to 0.8 , $p<0.001$ ). In subgroup analyses, we found that such an inverse association was present only among patients who were not eligible to receive erythropoietin therapy (ie, serum creatinine $\leqq 6 \mathrm{mg} / \mathrm{dL}$ and/or haematocrit value $\geqq 28 \%$ ).

Conclusions: Patients who received non-AA prescribed CHMs after the diagnosis of CKD, yet before the start of erythropoietin therapy had a lower risk of mortality than those who did not.

\section{INTRODUCTION}

Chronic kidney disease (CKD) is a common chronic disease with an estimated global

\section{Strengths and limitations of this study}

- This is the first large-scale population-based follow-up study to evaluate the relationship between the use of non-aristolochic acid (AA) prescribed Chinese herbal medicines (CHMs) after the diagnosis of chronic kidney disease (CKD) and the risk of mortality in patients with CKD.

- The major limitations of the study were similar to those inherent of a retrospective follow-up study.

- Other study limitations included the difficulty in identifying specific ingredients of non-AA prescribed CHMs that might be beneficial to patients with CKD, the lack of information on non-prescribed CHMs and renal function indices, and the unavailability of lifestyle factors that could potentially confound the relationship between the use of non-AA prescribed CHMs and the risk of mortality.

prevalence of approximately $8-16 \% .{ }^{1}$ CKD is also one of the main causes that contribute to the increasing mortality rate around the world. $^{2}$ Taiwan has the highest incidence of CKD and end-stage renal disease (ESRD) worldwide $^{3}$ and the use of Chinese herbal medicines (CHMs) is very popular in Taiwan. Previous studies had found that CHMs containing aristolochic acid (AA), a carcinogenic substance to human beings, were correlated with acute renal failure ${ }^{4}$ and urinary tract carcinoma. ${ }^{5}$ A large population-based study had also shown that the intake of any kinds of CHM was associated with the occurrence of CKD in Taiwan. ${ }^{6}$ However, in a recently published case-control study, the occurrence of CKD was found to be limited to participants who received non-prescribed CHMs, but not those who consumed prescribed CHMs. ${ }^{7}$ Moreover, some commonly prescribed CHMs such as Astragalus membranaceus (huang qi), Angelica sinensis (dong quai) 
and Rheum palmatum (da huang, rhubarb root) were suggested to possess renoprotective effects. ${ }^{8}$ Given that the association between the use of prescribed CHMs and the occurrence and/or disease progression of CKD remains unclear, we conducted a nationwide populationbased follow-up study to better understand the use of non-AA prescribed CHMs after the diagnosis of CKD and the effect of such medication use on the risk of mortality in patients with CKD.

\section{MATERIALS AND METHODS}

\section{Study population}

This retrospective follow-up study employed data derived from the National Health Insurance Research Database (NHIRD) in Taiwan to identify the study population. The NHIRD was established in 1996 and became more comprehensive in terms of data compilation in 1997. This database contains more than $96 \%$ of the entire Taiwanese population's medical information, including outpatient and emergency department visits, hospitalisation, medication use (conventional medicines and CHMs) and diagnostic data (coded by International Classification of Diseases, Ninth Revision, Health Problems, ICD-9-CM). ${ }^{9}$ The database used for this study is the longitudinal health insurance database randomly sampled by the Taiwan NHI Bureau in 2000 (Longitudinal Health Insurance Database 2000; LHID2000), which consists of one million Taiwanese population who were insured in that year. The LHID2000 contained follow-up medical information, spanning from 1997 to 2008, for the aforementioned cohort. The sampling method of the LHID2000 was completely random; and the distribution of age, gender and other characteristics of the sampled cohort was validated by the Taiwan National Health Research Institutes. Moreover, hundreds of studies utilising this database had been published in peer-reviewed journals and the database was believed to be comprehensive and reliable. ${ }^{11}$

\section{Eligible participants and duration of follow-up}

Within the LHID2000, we identified patients with CKD by searching for the following ICD-9-CM codes: 581-583, 585-589, 250.4, 274.1, 403 and 404. When a patient had at least three consecutive outpatient visits (equivalent to approximately 3 months of follow-up) or a hospitalisation record for the aforementioned ICD-9-CM codes, they were defined as a patient with CKD. We selected the aforementioned ICD codes after consulting a group of nephrologists to better understand the practice of coding CKD diagnosis in the NHIRD and taking into account the definition of CKD in previously published papers that also employed the NHIRD. ${ }^{12-14}$ To ensure that there was no pre-existing CKD and there was sufficient time (at least 3 years) to assess the participants' comorbidities and medication use prior to the diagnosis of CKD, we included only participants who had a firsttime diagnosis of CKD on or after 1 January 2000.
Moreover, all participants must be diagnosed with CKD prior to 31 December 2007 to ensure a follow-up time of at least 1 year.

Because AA-containing CHMs were proven to cause acute renal toxicity and urothelial cancer, ${ }^{4-6}$ we excluded participants who had taken any of the following AA-containing medications before the diagnosis of CKD: Ma-Dou-Ling (Fructus aristolochiae), Tian-Xian-Teng (Caulis aristolochiae), Xi-Xin (Asarum heterotoppoides), Guan-Mu-Tong (Aristolochia manshuriensis), Guang-Fangchi (Aristolochia fangchi), Qing-Mu-Xiang (Radix aristolochiae), Mu-Tong (Akebia spp), Fangchi (Stephania spp) and Mu-Xiang (Radix aucklandiae) ${ }^{5}{ }^{15}$ Participants were also excluded if they were diagnosed with cancer prior to the diagnosis of CKD, had HIV infection and were not Taiwanese citizens. Finally, because patients who died within 1 month after the diagnosis of CKD were found to be less likely to receive non-AA prescribed CHMs (data now shown) probably because they had a more severe underlying disease or were even in a moribund state, we excluded such participants to minimise the possibility of selection bias.

All eligible participants were then followed from the time of incident diagnosis of CKD until death or 31 December 2008, whichever came first. The outcome of interest was the risk of all-cause mortality in patients with CKD during the follow-up period. Death was defined as withdrawal of the patients with CKD from the NHI programme.

\section{Assessment of CHM use}

All CHMs prescribed by certified Chinese medicine practitioners and manufactured following 'Good Manufacturing Practice' are eligible to be reimbursed by the NHI Bureau in Taiwan and one cannot purchase prescribed CHMs in Taiwan without a certified Chinese medicine practitioner's prescription. Therefore, the LHID2000 contained all information on prescribed CHMs, and we were able to assess the participants' overall use of prescribed CHMs during the study period, including the names and the amount of prescribed CHMs. ${ }^{16}$ Moreover, according to the standard prescriptions recommended by the Committee of Chinese Medicine and Pharmacy (CCMP) in Taiwan, the ingredients of each compound CHM could also be identified. ${ }^{17}$ After the incident diagnosis of CKD, each participant's use of non-AA prescribed CHMs, including the date, amount and duration of each prescription was assessed. Owing to the complexity of prescribed CHMs, which could comprise either a single ingredient or a compound product (ie, a mixture of different CHM ingredients) and the prescribed medications could be manufactured in powder, liquid, tablet or other forms, the cumulative exposure dose of individual CHMs was extremely difficult to determine. Therefore, we eventually divided the use of non-AA prescribed CHMs into non-use, use only prior to the diagnosis of CKD, use only after the diagnosis of CKD and use in both periods before and after the diagnosis of CKD. 
Assessment of potential confounders

To control for the effects of potential confounders, we obtained the data on age, gender, comorbidities, prior hospitalisation, concomitant use of non-steroidal antiinflammatory drugs (NSAIDs) and other analgesics (including acetaminophen, buprenorphine, codeine, meperidine, morphine, nalbuphine and tramadol that were reimbursed by the Taiwan NHI Bureau during the study period), and the receipt of erythropoietin therapy within the LHID2000 database. We classified age into three groups, that is, less than or equal to 40, 40-65 and greater than 65 years. For pre-existing comorbidities, we paid special attention to hypertension, diabetes mellitus and cardiovascular disease (CVD) (including congenital heart disease) and ascertained relevant diagnoses by searching for three consecutive outpatient visits and/or any hospitalisation for the ICD-9 codes of individual diseases within 1 year before the incident diagnosis of CKD. We also employed Charlson's Comorbidity Index $(\mathrm{CCI})^{18}$ to control for the overall disease burden of various comorbidities on each study participant. The definitions of comorbidities other than hypertension, diabetes mellitus and CVD were similar to those of the aforementioned diseases. We further defined participants who were hospitalised within 1 year prior to the diagnosis of CKD as having any 'hospitalization before the diagnosis of CKD'.

In addition to comorbidities, we assessed the participants' overall medication history, including the date, amount and duration of prescriptions before and after the diagnosis of CKD. ${ }^{16}$ We specifically examined the use of NSAIDs, analgesics and erythropoietin, which were likely to confound the relationship between the use of non-AA prescribed CHMs and the risk of mortality in patients with CKD. We divided the use of NSAIDs and analgesics by the annual total amount of pills received by the study participants within the previous year into $\leqq 104$ and $>104$ pills. ${ }^{19}$ The receipt of erythropoietin, a surrogate marker of the severity of CKD in Taiwan because its use was strictly limited to patients with ESRD who had a haematocrit (Hct) value less than $28 \%$ and/ or patients with CKD who had a serum creatinine greater than $6 \mathrm{mg} / \mathrm{dL}$ and a Hct value less than $28 \%$, was classified as use or non-use.

\section{Statistical analyses}

We employed log-rank test and Holm-Sidak multiple comparisons to examine the survival rate between patients with different patterns of non-AA prescribed CHM use before and after the diagnosis of CKD. We further performed Cox proportional hazard regression to assess the association between various predictors and study participants' survival rates. We also conducted subgroup analyses by stratifying on the surrogate marker of the severity of CKD, that is, the receipt of erythropoietin therapy, to see whether the severity of CKD would modify the effect between non-AA prescribed CHMs and the risk of mortality. Moreover, we performed sensitivity analysis by excluding participants whose CKD was defined by ICD codes other than 585 (chronic renal failure) and 586 (renal failure, unspecified). All variables with a $p$ value less than 0.05 in univariate analyses were entered into the multivariate analyses. All statistical analyses were performed with the SAS statistical package V.9.1.3 (SAS Institute, Inc, Cary, North Carolina, USA).

\section{RESULTS}

A total of 47876 participants had an incident diagnosis of CKD after 1 January 2000. Among them, 32000 had ever used AA-containing prescribed CHMs and were thus excluded. We further excluded 60 participants who were not Taiwanese citizens and 1882 participants with any type of cancer and/or HIV infection before the diagnosis of CKD. Seventy participants who died within the first month of CKD diagnosis were excluded as well. This resulted in a total of 13864 participants eligible for final analysis.

The study participants had a mean age of 54.2 \pm 20.9 years. A total of 929 participants $(6.7 \%)$ died during the follow-up period between 2000 and 2008. In univariate analyses, the mortality rate was higher in men as compared with women. Older participants also had a higher mortality rate than the younger ones. Moreover, participants who had a higher CCI score, were hospitalised within 1 year prior to the diagnosis of CKD and were diagnosed with hypertension, diabetes mellitus and CVD, were found to have a higher mortality rate. The receipt of greater than 104 pills of NSAIDs and/or analgesics annually, and the treatment with erythropoietin were associated with a high risk of mortality as well.

Use of non-AA prescribed CHMs was fairly common among patients with CKD with $25.1 \%$ of the survivors and $14.4 \%$ of the deceased received non-AA prescribed CHMs after the diagnosis of CKD (table 1). The crude mortality rate appeared to be highest among those who never received non-AA prescribed CHMs (ie, non-use), followed by those who received non-AA prescribed CHMs only prior to the diagnosis of CKD, those with non-AA prescribed CHMs use only after CKD, and those with prescribed CHMs use before and after the diagnosis of CKD. The observed trend between different groups of non-AA prescribed CHMs use was statistically significant (table 1). The study findings further indicated that receipt of non-AA prescribed CHMs after the diagnosis of CKD, with or without the use of non-AA prescribed CHMs before CKD diagnosis, had a significantly better survival rate than those who never received or those who received non-AA prescribed CHMs only before the diagnosis of CKD ( $p<0.001$; figure 1$)$.

In the multivariate analysis that employed the non-use group as the reference, we found that the mortality rate was similar among those with non-AA prescribed CHMs use only before the diagnosis of CKD. By contrast, those participants who received non-AA prescribed CHMs only after the diagnosis of CKD had a lower risk of mortality 
Table 1 Distribution of baseline characteristics of 13864 patients with CKD in Taiwan

\begin{tabular}{|c|c|c|c|}
\hline Characteristics & Survivors (n, \%) & Deceased (n, \%) & p Value \\
\hline \multicolumn{4}{|l|}{ Gender } \\
\hline Female & $5011(38.7)$ & $326(35.1)$ & \multirow[t]{2}{*}{0.028} \\
\hline Male & 7924 (61.3) & $603(64.9)$ & \\
\hline \multicolumn{4}{|l|}{ Age group (years) } \\
\hline$<40$ & 3435 (26.6) & $28(3.0)$ & \multirow[t]{3}{*}{$<0.001$} \\
\hline $40-65$ & $5243(40.5)$ & $197(21.2)$ & \\
\hline$>65$ & 4257 (32.9) & $704(75.8)$ & \\
\hline \multicolumn{4}{|l|}{ Hypertension } \\
\hline No & 6969 (53.9) & $225(24.2)$ & \multirow[t]{2}{*}{$<0.001$} \\
\hline Yes & $5966(46.1)$ & $704(75.8)$ & \\
\hline \multicolumn{4}{|l|}{ Diabetes mellitus } \\
\hline No & $9241(71.4)$ & $492(53.0)$ & \multirow[t]{2}{*}{$<0.001$} \\
\hline Yes & $3694(28.6)$ & $437(47.0)$ & \\
\hline \multicolumn{4}{|l|}{ Cardiovascular disease* } \\
\hline No & $10114(78.2)$ & $581(62.5)$ & \multirow[t]{2}{*}{$<0.001$} \\
\hline Yes & 2821 (21.8) & $348(37.5)$ & \\
\hline \multicolumn{4}{|l|}{ Charlson comorbidity index } \\
\hline 0 & 3263 (25.2) & $108(11.6)$ & \multirow[t]{3}{*}{$<0.001$} \\
\hline 1 & $2798(21.6)$ & $143(15.0)$ & \\
\hline$\geq 2$ & $6874(53.1)$ & 678 (73.3) & \\
\hline \multicolumn{4}{|l|}{ Any hospitalisation before CKD† } \\
\hline No & 11939 (92.3) & $719(77.4)$ & \multirow[t]{2}{*}{$<0.001$} \\
\hline Yes & $996(7.7)$ & $210(22.6)$ & \\
\hline \multicolumn{4}{|c|}{ Receipt of NSAID >104 pills/year } \\
\hline No & $12764(98.7)$ & $904(97.3)$ & \multirow[t]{2}{*}{$<0.001$} \\
\hline Yes & $171(1.3)$ & $25(2.7)$ & \\
\hline \multicolumn{4}{|c|}{ Receipt of analgesics >104 pills/year } \\
\hline No & $12588(97.3)$ & $883(95.0)$ & \multirow[t]{2}{*}{$<0.001$} \\
\hline Yes & $347(2.7)$ & $46(5.0)$ & \\
\hline \multicolumn{4}{|l|}{ Receipt of erythropoietin } \\
\hline No & 12398 (95.9) & $832(89.6)$ & \multirow[t]{2}{*}{$<0.001$} \\
\hline Yes & $537(4.1)$ & $97(10.4)$ & \\
\hline \multicolumn{4}{|c|}{ Use of non-AA prescribed CHMs† } \\
\hline Non-use & $7343(56.8)$ & $643(69.2)$ & \multirow[t]{4}{*}{$<0.001$} \\
\hline Before CKD only & $2336(18.1)$ & $152(16.4)$ & \\
\hline After CKD only & $1943(15.0)$ & $83(8.9)$ & \\
\hline Both before and after CKD & $1313(10.1)$ & $51(5.5)$ & \\
\hline
\end{tabular}

${ }^{*}$ Cardiovascular diseases include congenital heart disease.

†CKD and CHMs denote (the diagnosis of) chronic kidney disease and Chinese herbal medicines respectively; Cochran-Armitage trend test $\mathrm{p}$ value $<0.001$.

AA, aristolochic acid; CHMs, Chinese herbal medicines; CKD, chronic kidney disease; NSAID, non-steroidal anti-inflammatory drug

in the follow-up period (adjusted HR (aHR) 0.6; 95\% CI 0.4 to $0.7 ; \mathrm{p}<0.001)$. Moreover, participants with non-AA prescribed CHMs use before and after the diagnosis of CKD also showed a lower mortality rate (aHR 0.6; 95\% CI 0.5 to $0.8 ; \mathrm{p}<0.001$; table 2 ).

In the subgroup analyses stratified by the receipt of erythropoietin therapy, we found that the inverse association between the use of non-AA prescribed CHMs and the risk of mortality was present only among patients who did not receive erythropoietin therapy (aHR 0.3 (95\% CI 0.3 to 0.4 ) for patients who received non-AA prescribed CHMs only after the diagnosis of CKD; and aHR 0.4 (95\% CI 0.3 to 0.5 ) for patients who received non-AA prescribed CHMs before and after the diagnosis of CKD). It is also noteworthy that the use of non-AA prescribed CHMs was not associated with a higher risk of mortality among patients who received erythropoietin therapy (data not shown).

In the sensitivity analysis that included only participants whose CKD diagnosis was defined by ICD codes 585 and/ or 586, the number of study participants decreased from 13864 to 7159; however, the inverse association between non-AA prescribed CHMs and all-cause mortality among patients with CKD remained statistically significant. For patients who received non-AA prescribed CHMs only before the diagnosis of CKD, the aHR was $0.9(95 \%$ CI 0.7 to 1.1$)$; whereas the aHR was $0.6(95 \%$ CI 0.5 to 0.8$)$ and 0.5 (95\% CI 0.3 to 0.7 ) for patients who received non-AA prescribed CHMs only after the diagnosis of CKD and for patients who received non-AA prescribed CHMs before and after CKD, respectively. 


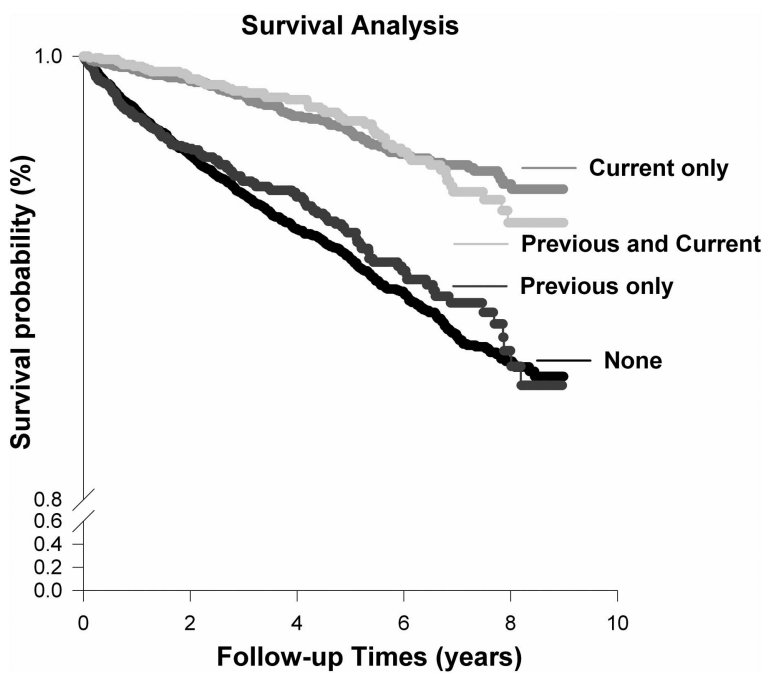

Figure 1 Survival curve of 13864 patients with chronic kidney disease (CKD), defined by their use of non-aristolochic acid (AA) prescribed Chinese herbal medicines (CHMs) before and after the diagnosis of CKD note: group $A$ : non-use of non-AA prescribed CHMs; group $B$ : use of non- $\mathrm{AA}$ prescribed $\mathrm{CHMs}$ only before the diagnosis of CKD; group C: use of non-AA prescribed CHMs only after the diagnosis of CKD; group D: use of non-AA prescribed CHMs before and after the diagnosis of CKD. Log-rank test (Holm-Sidak method): group A versus C, $p<0.001$; group $A$ versus $D, p<0.001$; group $B$ versus $C$, $p<0.001$; group $B$ versus $D, p<0.001$.

\section{DISCUSSION}

Compared with participants without CKD, the occurrence of cardiovascular events and the risk of mortality were higher among patients with CKD. ${ }^{20}$ Therefore, identifying possible treatments that can delay the progression of CKD is important in the prevention of mortality among patients with CKD. Although the use of CHMs was frequently associated with the risk of CKD, the exact relationship between the use of CHMs and the occurrence and/or progression of CKD remains unclear. In this study, we employed a nationwide population-based database to investigate the effects of non-AA prescribed CHMs on the risk of mortality in patients with CKD. We found that among patients with CKD, men, older age, the presence of certain comorbidities (ie, hypertension, diabetes mellitus and CVD), receipt of higher dose of analgesics, treatment with erythropoietin and hospitalisation prior to the diagnosis of CKD were associated with a higher risk of mortality; whereas the use of non-AA prescribed CHMs after the diagnosis of CKD yet before the start of erythropoietin therapy was related to a lower risk of mortality.

Many studies have investigated the association between CHMs use and the risk of CKD and/or ESRD, and there was plenty of evidence suggesting a likely causal relationship between the consumption of AA-containing CHMs and the development of acute renal failure, ${ }^{4}$ CKD, ESRD $^{13}$ and urothelial cancer. ${ }^{5}$ Studies that did not differentiate between AA-containing CHMs and non-AA CHMs thus might overestimate the adverse effects of non-AA CHMs. For example, Wen et at found a positive association between CHM use and the risk of CKD. However, it was unclear whether the observed association was attributable to AA-containing CHMs or other CHMs.

Table 2 Cox proportional hazard analysis of the predictors of all-cause mortality in 13864 patients with CKD in Taiwan

\begin{tabular}{|c|c|c|c|c|}
\hline Characteristics & Crude HR $(95 \% \mathrm{Cl})$ & $\mathrm{p}$ Value & Adjusted HR $(95 \% \mathrm{Cl})$ & p Value \\
\hline Gender (male) & $1.2(1.1$ to 1.4$)$ & 0.004 & $1.3(1.1$ to 1.4$)$ & 0.001 \\
\hline \multicolumn{5}{|l|}{ Age group (years) } \\
\hline$<40$ & Reference & - & Reference & - \\
\hline $40-65$ & 5.0 (3.3 to 7.4$)$ & $<0.001$ & $3.6(2.4$ to 5.4$)$ & $<0.001$ \\
\hline$>65$ & $5.0(4.1$ to 6.0$)$ & $<0.001$ & 3.7 (3.1 to 4.6$)$ & $<0.001$ \\
\hline Hypertension & 4.2 (3.6 to 4.9$)$ & $<0.001$ & $1.4(1.2$ to 1.6$)$ & $<0.001$ \\
\hline Diabetes mellitus & 2.4 (2.1 to 2.8$)$ & $<0.001$ & $1.3(1.1$ to 1.4$)$ & $<0.001$ \\
\hline Cardiovascular disease* & 2.5 (2.2 to 2.9$)$ & $<0.001$ & $1.2(1.0$ to 1.4$)$ & 0.024 \\
\hline \multicolumn{5}{|l|}{ Charlson comorbidity index } \\
\hline 0 & Reference & - & - & - \\
\hline 1 & $1.6(1.3$ to 2.1$)$ & $<0.001$ & & \\
\hline$\geq 2$ & 3.4 (2.8 to 4.2$)$ & $<0.001$ & & \\
\hline Any hospitalisation before CKD $\dagger$ & $4.3(3.7$ to 5.0$)$ & $<0.001$ & $2.6(2.2$ to 3.1$)$ & $<0.001$ \\
\hline Receipt of NSAID >104 pills/year & 3.1 (2.1 to 4.7$)$ & $<0.001$ & - & - \\
\hline Receipt of analgesics > 104 pills/year & $3.0(2.2$ to 4.1$)$ & $<0.001$ & $1.8(1.4$ to 2.5$)$ & $<0.001$ \\
\hline Receipt of erythropoietin & 2.4 (2.0 to 2.9 ) & $<0.001$ & 1.4 (1.1 to 1.8$)$ & 0.002 \\
\hline \multicolumn{5}{|l|}{ Use of non-AA prescribed CHMs† } \\
\hline Non-use & Reference & - & Reference & - \\
\hline Before CKD only & $0.9(0.8$ to 1.1$)$ & 0.286 & $1.0(0.8$ to 1.2$)$ & 0.739 \\
\hline After CKD only & $0.4(0.3$ to 0.5$)$ & $<0.001$ & $0.6(0.4$ to 0.7$)$ & $<0.001$ \\
\hline Both before and after CKD & $0.4(0.3$ to 0.5$)$ & $<0.001$ & $0.6(0.5$ to 0.8$)$ & $<0.001$ \\
\hline
\end{tabular}

${ }^{*}$ Cardiovascular diseases include congenital heart disease.

†CKD and CHMs denote (the diagnosis of) chronic kidney disease and Chinese herbal medicines, respectively.

AA, aristolochic acid; CHMs, Chinese herbal medicines; CKD, chronic kidney disease; NSAID, non-steroidal anti-inflammatory drug 
The inability to differentiate between AA-containing CHMs and non-AA CHMs had also resulted in the prejudicial term 'Chinese herbal nephropathy' and led many western medicine practitioners to warn the patients with CKD against the use of any CHMs. In this study, we noticed that nearly two-thirds of those patients who had used prescribed CHMs prior to the diagnosis of CKD discontinued the treatment after the diagnosis of CKD. However, not all CHMs were harmful. In fact, some animal experiments and clinical studies have shown that the use of certain CHMs could protect against the development of CKD or delay the deterioration of CKD. ${ }^{8}$ 21-24 In our previous study, we also found that CKD/ESRD was related to the use of non-prescribed CHMs, but not non-AA prescribed CHMs. ${ }^{7}$ The aforementioned findings suggested that non-AA CHMs, appropriately prescribed by certified Chinese medicine practitioners, could have a renoprotective effect or at least a non-nephrotoxic effect in patients with CKD.

In this study, we excluded all participants who had used AA-containing CHMs before the diagnosis of CKD to eliminate the likely strong confounding effects of AA. Our study results showed that patients with CKD who received non-AA prescribed CHMs after the diagnosis of CKD, with or without the use of non-AA prescribed CHMs prior to CKD, had a significantly lower risk of mortality than non-users or those who discontinued non-AA prescribed CHMs after the diagnosis of CKD. In the subgroup analyses stratified by the receipt of erythropoietin therapy, we further found that the above-noted inverse association was present only among patients whose CKD was not severe enough to warrant erythropoietin therapy. Given that the function of kidney exponentially declines as CKD progresses, the use of and the response to non-AA prescribed CHMs might be significantly different between patients with different stages of CKD. The findings of this study thus supported the proposition that non-AA CHMs prescribed by certified Chinese medicine practitioners might be helpful in delaying the progression of CKD and/or its complications (eg, CVD) in patients with earlier stages of CKD and subsequently reducing their risk of mortality.

In Taiwan, according to the unified formulas of CHMs provided by the Taiwanese CCMP, the majority of prescribed CHMs are compound (cocktail) formulas. ${ }^{17}$ The CHM formulas often contain 'renoprotective' CHM ingredients such as Astragalus membranaceus, Angelica sinensis and Rheum palmatum. These CHMs were found to inhibit renal tubulointerstitial fibrosis, ${ }^{24}$ enhance nitric oxide production and alleviate renal interstitial fibrosis, ${ }^{23}$ and lower serum cholesterol, blood urea nitrogen, creatinine, oxidative stress and profibrotic factors to attenuate nephritis and renal necrosis. ${ }^{8}$ Although there was no existing large-scale randomised controlled trials to support the beneficial effects of the aforementioned CHMs, our findings did indicate that the use of such non-AA prescribed CHMs might reduce the mortality risk in patients with CKD. Further studies are warranted to delineate the exact ingredients and mechanisms of non-AA prescribed CHMs that can delay the progression of CKD and/or its complications.

In addition to non-AA prescribed CHMs, we also found that male gender and older age were associated with a higher risk of mortality in patients with CKD, a finding consistent with previous reports. ${ }^{19}{ }^{20}$ Female sex hormones are known to inhibit inflammatory and apoptotic processes, which can then protect renal tissues. ${ }^{25}$ Therefore, female gender is considered as a protective factor in many kidney diseases and a lower mortality rate caused by CKD has been observed in women as compared with men. By contrast, older age and increased comorbidities have been associated with higher disease severity and mortality rate. ${ }^{26}$

In this study, several comorbidities were associated the risk of mortality in patients with CKD as well. Among them, hypertension is a well-known risk factor for the progression of CKD and abnormal blood pressure would cause the deterioration of CKD, which can lead to cardiovascular complications and mortality. ${ }^{27}$ Diabetes mellitus is also an important risk factor for the progression of CKD. According to previous reports, abnormal glycated haemoglobin can result in a higher probability of CVD as well as macroalbuminuria. These complications then lead to faster progression of CKD, or even death. ${ }^{28}$ Many literature reports had further indicated that CVD is the major complication as well as the cause of mortality in patients with CKD. ${ }^{29}$ Therefore, the observed association between hypertension, diabetes mellitus and CVD and a higher risk of mortality in patients with CKD seems plausible.

The risk of mortality was also related to the use of analgesics and erythropoietin and any hospitalisation prior to CKD diagnosis. The use of analgesics was believed to be related to CKD deterioration, ${ }^{19}$ and our finding on the use of analgesics did suggest such an association. Erythropoietin is a type of glycoprotein hormone, which is used in the regulation of haemoglobin. The use of erythropoietin therefore indicates a deficiency in the production of renal erythropoietin and suggests the need of dialysis or worst renal function in the recipients. Similarly, if a participant was hospitalised prior to the diagnosis of CKD, they were likely to be in a poor health condition. Both variables were therefore expected to be associated with a higher risk of mortality among patients with CKD, as evidenced in this study.

Some previous studies had shown that the use of NSAIDs was related to the deterioration and subsequent mortality in patients with $\mathrm{CKD}^{30}$; and the consumption of ultra-high cumulative dose of NSAIDs (ie, a lifetime dose of 5000 pills) was linked to CKD deterioration and mortality. ${ }^{30}$ However, some long-term studies did not show a direct relationship between NSAID use and the risk of renal function loss. ${ }^{31}{ }^{32}$ In this study, we found that, after controlling for other confounders, the relationship between NSAID use and the risk of mortality did not reach statistical significance. This could at least 
be partially explained by the fact that only very few study participants had consumed 5000 or more pills of NSAIDs during the follow-up period.

\section{LIMITATIONS}

There are several limitations of this study and a causal relationship between the use of non-AA prescribed CHMs and the risk of mortality cannot be inferred based on such an observational study. First, despite the fact that we selected only patients with incident CKD and assessed the effects of non-AA prescribed CHMs use primarily after the diagnosis of CKD to minimise the possibility of selection bias, unforeseen differences in baseline characteristics between patients who received and those who did not receive non-AA prescribed CHMs after the diagnosis of CKD could still exist and account for the study findings.

Second, although we employed Cox proportional hazard regression analysis to control for potential confounders, we did not have data on personal information such as lifestyle, smoking and ethanol drinking, which might confound the relationship between the use of non-AA prescribed CHMs and the risk of mortality.

Third, we did not have access to data on serum creatinine and/or other renal function indices in the NHIRD; therefore, the diagnosis of CKD in this study could only be defined by specific ICD codes. Although we have exercised great caution in defining the diagnosis of CKD, the possibility of coding error in the NHIRD could still be present and might result in an underestimate or overestimate of the relationship between non-AA prescribed CHMs and the risk of mortality. The inclusion of ICD codes not specific to CKD might also lead to a biased estimate. In the sensitivity analysis that limited the study participants to those whose CKD diagnosis was defined by ICD codes 585 and 586; we nevertheless found that the inverse association between non-AA prescribed CHMs and the risk of mortality remained statistically significant.

Fourth, although all CHMs prescribed by certified Chinese medicine practitioners in Taiwan are eligible to be reimbursed by the NHI Bureau, patients with CKD can still obtain non-prescribed CHMs by purchasing the herbs from the herbalists and/or uncertified Chinese medicine practitioners, or gathering CHMs of plant origin by themselves. While we did not have nationwide data on the use of non-prescribed CHMs in Taiwan, which might be hazardous to patients with $\mathrm{CKD}^{7}$ and associated with the use of prescribed CHMs, we did find in a previously published case-control study that only $6.6 \%$ of the controls received non-prescribed CHMs. ${ }^{7}$ Moreover, we did not find any significant relationship between the use of non-prescribed and prescribed CHMs in patients with CKD. Both findings suggested that the use of non-prescribed CHMs was less likely to significantly confound the inverse association between non-AA prescribed CHMs and the risk of mortality observed in this study.
Fifth, adulteration of CHMs by prescription drugs and heavy metals and contamination of CHMs by heavy metals have always been a concern in studying CHMs. In Taiwan, the NHI Bureau mandates all reimbursed CHMs must be manufactured following the 'Good Manufacturing Practice'. Moreover, the Taiwan Food and Drug Administration (FDA) has conducted annual screening of adulteration of CHMs by prescription drugs and periodically measured the concentrations of various heavy metals in raw materials of CHMs. According to the data reported by the Taiwan FDA, the possibility of adulteration of prescribed CHMs by prescription drugs and/or contamination of prescribed CHMs by heavy metals is negligible in Taiwan. ${ }^{33}$

Sixth, coding error of prescribed CHMs is possible in a large database like the NHIRD. Although we were unable to check the accuracy of the use of non-AA prescribed CHMs, the information was likely to be accurate and complete. In Taiwan, every CHM prescription is strictly regulated and only recorded prescriptions are eligible to receive reimbursement from the Taiwan NHI Bureau.

Seventh, because there were numerous types of analgesics and NSAIDs and we did not have information on over-the-counter analgesics/NSAIDs, the total number of analgesic/NSAID pills received by patients with CKD in this study might not fully capture the exact dose-response relationship between analgesics/NSAIDs and the risk of mortality in patients with CKD. Still, we did find a positive association between the use of analgesics and the risk of mortality in the multivariate analysis.

Finally, because the types of non-AA prescribed CHMs are extremely diverse, we did not examine the specific ingredients of various compound CHM formulas. Thus, we were unable to identify the specific non-AA prescribed CHM (eg, Angelica sinensis and Astragalus membranaceus) that was associated with reduced risk of mortality in patients with CKD. Moreover, the lack of information on the cumulative dose of each non-AA prescribed CHM prohibited us from conducting further evaluation of any dose-response relationship between specific prescribed CHMs and the risk of mortality.

In conclusion, we found that the use of non-AA prescribed CHMs after the diagnosis of CKD yet before the start of erythropoietin therapy was related to a lower risk of mortality. Further prospective studies are warranted to see whether such an association is truly causal and to identify the specific CHMs that may be beneficial to patients with CKD. Laboratory analysis of the prescribed CHMs to identify exactly which CHMs are in each compound formulation may also be needed. Moreover, it is of great interest to evaluate whether there will be any herb-drug interactions between the use of non-AA prescribed CHMs and western medicine among patients with CKD.

Author affiliations

${ }^{1}$ Institute of Environmental and Occupational Health Sciences, School of Medicine, National Yang-Ming University, Taipei, Taiwan 
${ }^{2}$ Department of Medical Education and Research, Taiwan Landseed Hospital, Tao-Yuan, Taiwan

${ }^{3}$ Institute of Public Health, School of Medicine, National Yang-Ming University, Taipei, Taiwan

${ }^{4}$ Division of Nephrology, Department of Medicine, Taiwan Landseed Hospital, Tao-Yuan, Taiwan

${ }^{5}$ Department of Chinese Medicine, Taiwan Landseed Hospital, Tao-Yuan, Taiwan

${ }^{6}$ Department and Graduate Institute of Health Care Management, Chang Gung University, Tao-Yuan, Taiwan

${ }^{7}$ Division of Clinical Toxicology \& Occupational Medicine, Department of Medicine, Taipei Veterans General Hospital, Taipei, Taiwan

Contributors CFH, SLH, CCC and CCY conceived of the study. CFH and CCY conducted the analyses. CCY led the writing of the manuscript. All authors commented on the analytic plan and interpretation and contributed to the editing and final approval of the manuscript.

Funding This work was supported by a grant (LS-2012-09) from Taiwan Landseed Hospital.

Competing interests None.

Ethics approval The study protocol was approved by the Landseed hospital institutional review board (IRB-12-23)

Provenance and peer review Not commissioned; externally peer reviewed.

Data sharing statement The dataset of this study was obtained from Taiwan National Health Insurance Research Database.

Open Access This is an Open Access article distributed in accordance with the Creative Commons Attribution Non Commercial (CC BY-NC 3.0) license, which permits others to distribute, remix, adapt, build upon this work noncommercially, and license their derivative works on different terms, provided the original work is properly cited and the use is non-commercial. See: http:// creativecommons.org/licenses/by-nc/3.0/

\section{REFERENCES}

1. Jha V, Garcia-Garcia G, Iseki K, et al. Chronic kidney disease: global dimension and perspectives. Lancet 2013;382:260-72.

2. Lozano R, Naghavi M, Foreman K, et al. Global and regional mortality from 235 causes of death for 20 age groups in 1990 and 2010: a systematic analysis for the Global Burden of Disease Study 2010. Lancet 2012;380:2095-128.

3. Burrows NR, Li Y, Williams DE. Racial and ethnic differences in trends of end-stage renal disease: United States, 1995 to 2005. Adv Chronic Kidney Dis 2008;15:147-52.

4. Vanherweghem JL, Depierreux M, Tielemans C, et al. Rapidly progressive interstitial renal fibrosis in young women: association with slimming regimen including Chinese herbs. Lancet 1993;341:387-91.

5. Lai MN, Wang SM, Chen PC, et al. Population-based case-control study of Chinese herbal products containing aristolochic acid and urinary tract cancer risk. J Natl Cancer Inst 2010;102:179-86.

6. Wen CP, Cheng TY, Tsai MK, et al. All-cause mortality attributable to chronic kidney disease: a prospective cohort study based on 462293 adults in Taiwan. Lancet 2008;371:2173-82.

7. Hsieh CF, Huang SL, Chen CL, et al. Increased risk of chronic kidney disease among users of non-prescribed Chinese herbal medicine in Taiwan. Prev Med 2012:55:155-9.

8. Wojcikowski K, Johnson DW, Gobe G. Herbs or natural substances as complementary therapies for chronic kidney disease: ideas for future studies. J Lab Clin Med 2006;147:160-6.

9. National Health Research Institutes. National Health Insurance Research Databases. http://w3.nhri.org.tw/nhird//date_01.html (accessed 13 Aug 2013).

10. Gau CS, Chang IS, Lin Wu FL, et al. Usage of the claim database of national health insurance programme for analysis of cisapride-erythromycin co-medication in Taiwan. Pharmacoepidemiol Drug Saf 2007;16:86-95

11. Lee EK, Cham TM, Tseng PL. Using the pharmacoepidemiology approach to evaluate the first-year posttransplantation ambulatory health care cost from the Longitudinal Health Insurance Database (2001 to 2006) in Taiwan. Transplant Proc 2010;42:957-60.

12. Kuo HW, Tsai SS, Tiao MM, et al. Epidemiological features of CKD in Taiwan. Am J Kidney Dis 2007;49:46-55.

13. Lai MN, Lai JN, Chen PC, et al. Increased risks of chronic kidney disease associated with prescribed Chinese herbal products suspected to contain aristolochic acid. Nephrology (Carlton) 2009;14:227-34.

14. Kuo HW, Tsai SS, Tiao MM, et al. Analgesic use and the risk for progression of chronic kidney disease. Pharmacoepidemiol Drug Saf 2010;19:745-51.

15. Food and Drug Administration, U.S. Aristolochic Acid: Listing of Botanical Ingredients of Concern. http://www.fda.gov/Food/ RecallsOutbreaksEmergencies/SafetyAlertsAdvisories/ucm095283. htm (accessed 20 Nov 2013).

16. Bureau of National Health Insurance, Ministry of Health and Welfare. Query of Drug reimbursed by National Health Insurance, 2012 [in Chinese]. http://www.nhi.gov.tw/query/query1.aspx? menu=21\&menu_id=713\&webdata_id=3510\&WD_ID=851 (accessed 16 Aug 2013)

17. Committee on Chinese Medicine and Pharmacy, Ministry of Health and Welfare. Unified formulas of Chinese Herbal Medicine. http:// www.mohw.gov.tw/CHT/DOCMAP/DM1.aspx?f_list_no=758\&fod list no=3918 (accessed 29 Jan 2014)

18. Charlson ME, Pompei $\mathrm{P}$, Ales $\mathrm{KL}$, et al. A new method of classifying prognostic comorbidity in longitudinal studies: development and validation. J Chronic Dis 1987;40:373-83.

19. Perneger TV, Whelton PK, Klag MJ. Risk of kidney failure associated with the use of acetaminophen, aspirin, and nonsteroidal antiinflammatory drugs. N Engl J Med 1994;331:1675-9.

20. K/DOQI clinical practice guidelines for chronic kidney disease: evaluation, classification, and stratification. Am J Kidney Dis 2002;39:S1-266.

21. Peng A, Gu Y, Lin SY. Herbal treatment for renal diseases. Ann Acad Med Singapore 2005;34:44-51.

22. Li Y, Shen L, Chen R, et al. Effects of Salivae Miltiorrhizae Liguspyragine Hydrochloride and Glucose Injection on the levels of main platelet thrombin receptors in chronic haemodialysis patients. Chin J Integr Med 2011;17:625-30.

23. Meng L, Qu L, Tang J, et al. A combination of Chinese herbs, Astragalus membranaceus var. mongholicus and Angelica sinensis, enhanced nitric oxide production in obstructed rat kidney. Vascul Pharmacol 2007;47:174-83.

24. Meng LQ, Tang JW, Wang Y, et al. Astragaloside IV synergizes with ferulic acid to inhibit renal tubulointerstitial fibrosis in rats with obstructive nephropathy. Br J Pharmacol 2011;162:1805-18.

25. Grzegorczyk K, Krajewska M, Weyde W, et al. Gender and kidney diseases: the clinical importance and mechanisms of modifying effects. Postepy Hig Med Dosw (Online) 2011;65:849-57.

26. Rodriguez-Puyol D. The aging kidney. Kidney Int 1998;54:2247-65.

27. Ravera M, Re M, Deferrari L, et al. Importance of blood pressure control in chronic kidney disease. J Am Soc Nephrol 2006;17: S98-103.

28. Fioretto $\mathrm{P}$, Bruseghin $\mathrm{M}$, Berto I, et al. Renal protection in diabetes: role of glycemic control. J Am Soc Nephrol 2006;17:S86-9.

29. Go AS, Chertow GM, Fan D, et al. Chronic kidney disease and the risks of death, cardiovascular events, and hospitalization. $N$ Engl $J$ Med 2004;351:1296-305.

30. Gooch K, Culleton BF, Manns BJ, et al. NSAID use and progression of chronic kidney disease. Am J Med 2007;120:280-7.

31. Kurth T, Glynn RJ, Walker AM, et al. Analgesic use and change in kidney function in apparently healthy men. Am J Kidney Dis 2003;42:234-44.

32. Curhan GC, Knight EL, Rosner B, et al. Lifetime nonnarcotic analgesic use and decline in renal function in women. Arch Intern Med 2004;164:1519-24.

33. Chen YH, Lu FL, Liu YC, et al. Survey on heavy metals in raw materials of traditional Chinese medicine. Ann Rep Food Drug Res 2012;3:373-84 